\title{
Fabrication of polymer compound microlens by lens-on-lens microstructures
}

\author{
Zehua $\mathrm{Xia}^{1}$,Yan $\mathrm{Li}^{1,{ }^{*}}$,Xiaoya $\mathrm{Su}^{1}$, Yanhua $\mathrm{Han}^{1}$,Zhongyi Guo ${ }^{2}, \mathrm{Jianmin}_{\mathrm{Gao}}{ }^{3}$, \\ Qiaoqun Sun ${ }^{4}$ and Shiliang $\mathrm{Qu}^{1}$ \\ ${ }^{1}$ Department of Optoelectronics Science, Harbin Institute of Technology at Weihai, Weihai, China \\ ${ }^{2}$ School of Computer and Information, Hefei University of Technology, Hefei, China \\ ${ }^{3}$ School of Energy Science and Engineering, Harbin Institute of Technology, Harbin, China \\ ${ }^{4}$ College of Power and Energy Engineering, Harbin Engineering University, Harbin, China \\ *E-mail: yanli@hitwh.edu.cn
}

\begin{abstract}
We proposed a novel method for fabricating polymer compound microlenses (PCMLs) using micro-inkjet technique and subsequent curing process. Two different types of PCMLs with sandwich microstructure (PDMS-Glycerol-PDMS), concave and convex PCMLs, have beendesigned and fabricated in experiments. Convex PCML has two real images and two foci. The concave PCML has one real and one virtual focal planes, whichcan generate one real image and one virtual image respectively. Moreover, the diameter of concave PCML can be controlled by adjusting the curing time and temperature. The proposed method is simple, efficient and suitable for realizing large-scale high numerical aperture PCMLs array, which has potential applications in diverse optical systems such as optical storage and three-dimensional imaging.
\end{abstract}

Keywords:Polymer compound microlens; Multilayer structure; Microstructure.

\section{Introduction}

In recent years, microlenses with multiple focal lengths play an important role in three-dimensional imaging and the real-time detection of unconfined or fluctuating targets. Microlenses with special structures have played more important roles than simple lenses in many applications, such as achieving illumination uniformity by Fresnel microlens[1], improving coupling efficiency of high-power laser diodes and single-mode fiber by an elliptic cone-shaped microlens[2], achieving two focal plane by polydimethylsiloxane (PDMS) lens-on-lens microstructure[3].Due to the low cost and easy formability, polymers are widely used to fabricate microlens[4, 5]. Many researchers unceasingly explores in this domain, the methods for fabricating the microlens are gradually matured, such as photo-resist reflow method[6, 7], gray-scale photolithography[8], micro-inkjet fabrication[9, 10], and femtosecond laser direct writing[3, 11, 12].Moreover, the laser direct writing technology canalso be used to fabricate convex microlenses, but there are some problems in surface quality, profile and fabricating efficiency, which should be improved[13, 14]. We have also noticed that the approaches mentioned above are multistep 
process and inefficient, which are not suitable for the fabrication of the lens with compound microstructure. Therefore, it is still a challenge to develop a high-efficient and low-cost strategy for fabricating the PCML array.

In this paper, based on the micro-inkjet technique, we proposed a simple method for fabricating PCMLs array with excellent multiple imaging abilities. Two different microstructures, includingconcave PCML and convex PCML,have beenfabricated successfully.The fabricated PCML issandwich microstructure (PDMS-Glycerol-PDMS), in which the middle layer (glycerol) can be used to modulate the surface profile of the PCML. For realizing the PCMLs with different surface profile, the substrates should be placed in different way tosolidifiedPDMS. The solidification temperature and time should also be optimized to realize PCML with desired diameter. Experimental results show that the fabricated convex PCML has two real images, and the concave PCML has one real image and one virtual image due to the central concave surface. The topsmall lensdiameter of PCML can be controlled by adjusting the solidification temperature and curing time. The fabricated PCMLs array can also be fabricated with potential applications in optical storage[15, 16], extended depth of focus of a laser beam[17], real-time detection of the unconfined or fluctuating targets[18].

\section{Experimental}

The fabrication process of the PCML is shown in figure 1. Commercial silica glass was chosen as the base material, on which thin Teflon film was prepared by spin-coating method. The speed of the Vacuum Spin Coater (MTI VTC-100) was 1500 rad/min and the spin time was 60 seconds, respectively. Then thin Teflon film was put into a tube furnace annealing at $380^{\circ} \mathrm{Cfor} 10$ minutes. Next, in step II, glycerol droplets were produced by a syringe ( $2.5 \mu \mathrm{l}$, Eppendorf) with precision of $0.1 \mu \mathrm{l}$. As shown in figure 1 , the glycerol droplets array can be formed on the top of Teflon film and the distance between adjacent droplets can be controlled by a three-dimensional stage (Prior Scientific Inc.) with precision of 100nm. In step III, the glycerol droplets were covered with PDMS droplets as encapsulation layer and outer lens.The schematic compound structure is shown in step III, in which the glycerol droplet is wrapped by PDMS droplet. For guaranteeing precision, the needle of the syringe mustbe as close as possible to the glycerol droplets. Because of the large contact angle (as shown in figure1)of glycerol droplet against Teflon film, it is easy to realize the suspended glycerol droplet in the PDMS by inverting the substrate over 40 minutes. Then the 
PDMS wassolidified for realizing the PCMLs. However, in the step IV, there are two ways to solidify the PDMS as shown in figure 1 for fabricating convex PCML and concave PCML. Normal solidification (PDMS droplets on the top of glass)can form concave PCML.Inverted solidification (PDMS droplets on the bottom of glass), can form convex PCML.

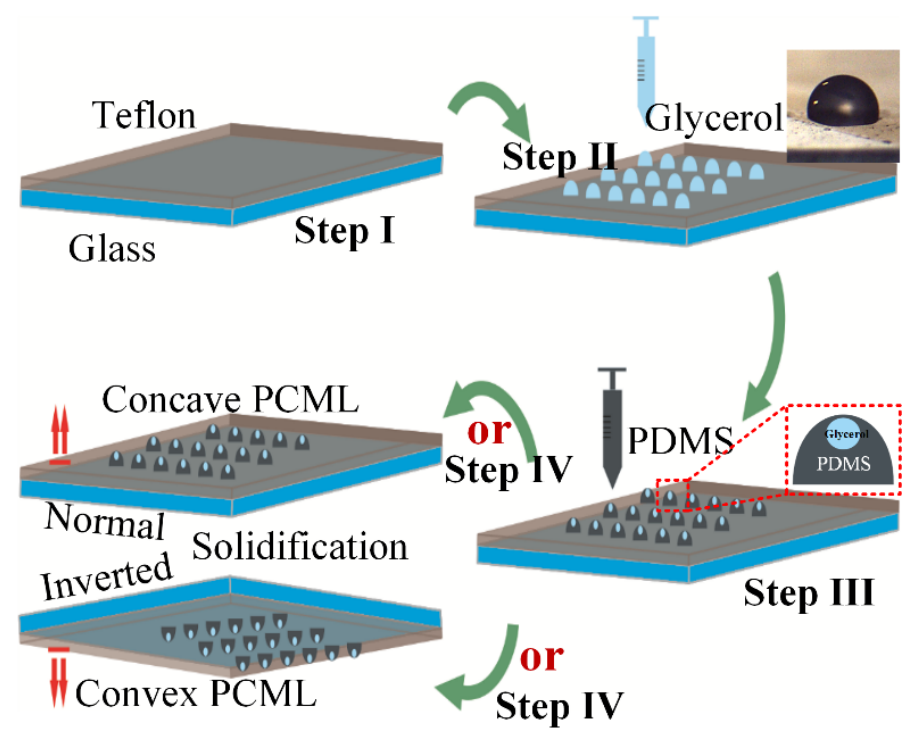

Figure 1.The schematic diagram of the fabricating process.

In the inverted solidification process, the slow evaporation of the glycerol leads to concave surface of the PDMS droplet. As shown in figure 2(a), the outer PDMS flows into the concave bottom and condenses into a smaller PDMS droplet with spherical surface due to gravity and surface tension. Because of the poor fluidity of PDMS, for generating convex PCML, the solidification spentmore time at low temperature. The fabricated convex PCML is shown infigure 2(c). The fabricated convex PCML shows clear interface between glycerol and PDMS.The convex PCML was solidified at $90^{\circ} \mathrm{C}$ for 50 minutes. Since the evaporation of glycerol leads to concave surface, the concave PCMLcan be realizedby normal solidification with the droplets on the top of substrates.As shown in figure 2(b), during the solidification process, the high temperature leads to evaporation of glycerol and progressively smaller glycerol droplet results in concave surface. Andthe PDMS cannot flow into the top concave surface because of gravity. That is the solidification temperature and time relate to the size of concave surface.Using the normal solidification, the fabricated concave PCML can be seen in figure 2(d) with clear interface between glycerol and PDMS.The concave PCML was solidified at $110^{\circ} \mathrm{C}$ for 20 minutes. Therefore, the PCML with concave or convex 
surface can be fabricated by choosing different solidification method.From figure 2(c) and figure 2(d) we can see that the sandwich microstructure of PCML can be considered as a new kind of polymer triplet microlenses (PDMS-Glycerol-PDMS).

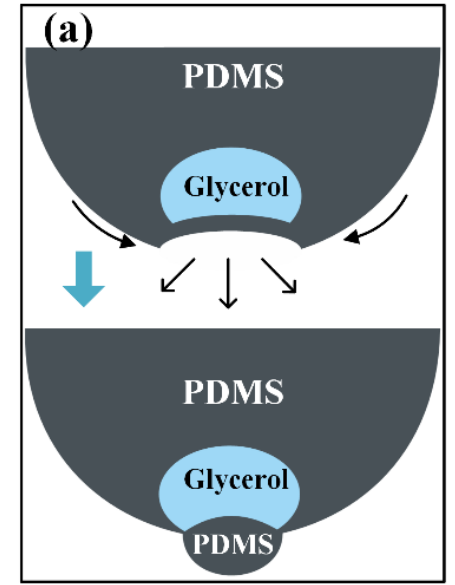

(c)

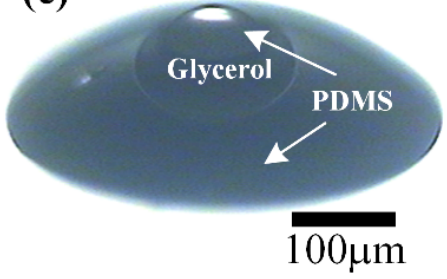

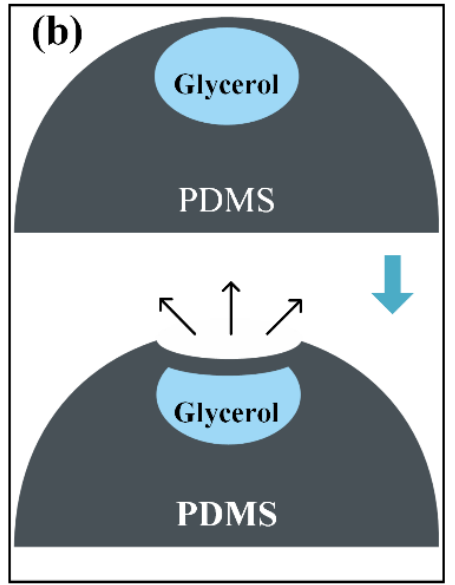

(d)

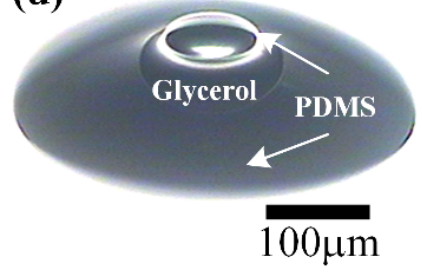

Figure 2.The schematics of convex surface (a) and concave surface (b)PCMLs. The fabricated PCML with convex surface (c) and concave surface (d).

\section{Results and discussion}

By using micro-inkjet technique, PCMLs array were fabricated with $1 \mathrm{~mm}$ interval between single PCML. For characterizing the imaging ability of the PCMLs array, a letter "F" printed in a paper was placed in front of the PCMLs array, and a CCD camera was used behind the PCMLs array to capture the images. By modulating the position of the CCD camera, images can be observed. As shown in figure3(a) and figure3(b),there are two real images in different focal plane generated by convex PCMLs array.Theseimages indicate that the convex PCMLs array have excellent imaging ability that has potential applications such as optical storage, three-dimensional image and real-time detection.Moreover, we also investigated the imaging abilities of the concave PCMLs array. As shown in figure3(c) and figure3(d), the inverted images and erect imagescan be obtained which represent the virtual focus imaging ability and real 
focus imaging ability. The experimental results indicate that the concave PCMLs array also have excellent imaging ability. But, from the images array, we can see that the uniformity of the PCMLs is still a problem by using the micro-inkjet technique. PDMS and glycerol are viscous liquid, it is still difficult to transmission under high pressure. Therefore, how to control the volume of glycerol and PDMS precisely is the key point in preparing PCMLs array.

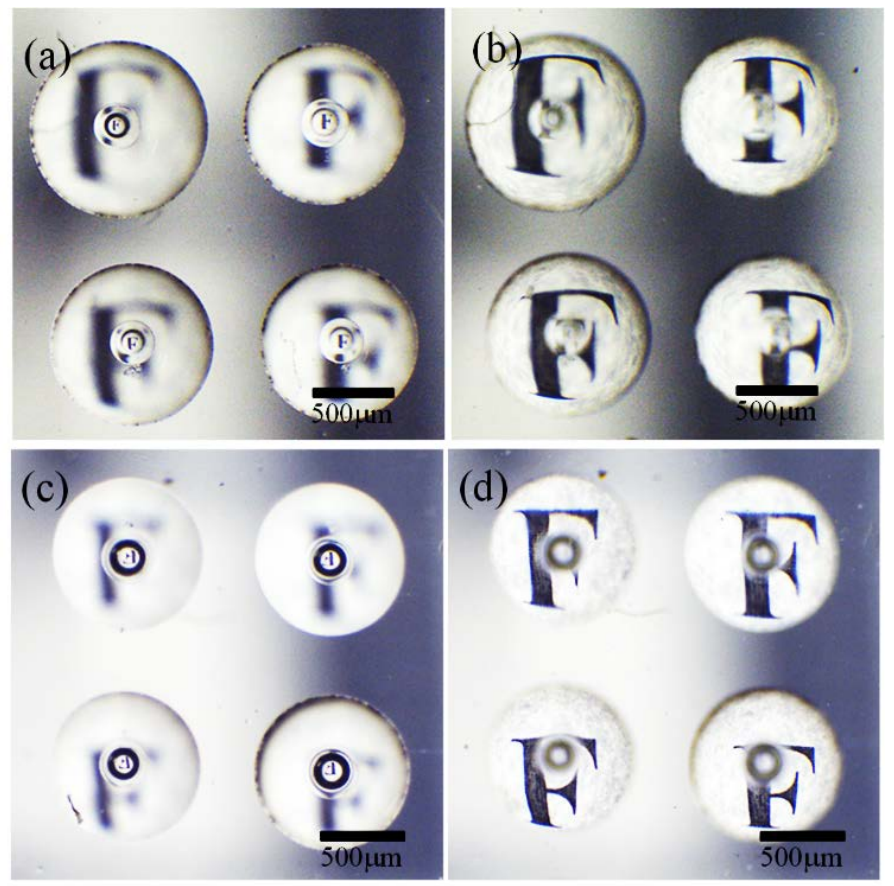

Figure 3.Experimental results of PCMLs array imaging ability, (a) and (b) are the first and second real images of convex PCMLs array, (c) and (d) are the virtual and real images of concave PCMLs array.

For investigating the relationship between curing temperature and top surface profile, PCMLs were fabricated with the same curing time (30 minutes) and different curing temperature, as shown in figure4. When the curing temperature were $80^{\circ} \mathrm{C}$ and $90^{\circ} \mathrm{C}$, the convex PCMLs can be realized, as shown in Fig. 4(a) and figure4(b). The obtained central erect images proved the top convex surface. However, with the temperature increasing, the top surface of PCML trends toward a plane surface as shown in Fig. 4(c) whose curing temperature was $100^{\circ} \mathrm{C}$. When the curing temperature reaches to $110^{\circ} \mathrm{C}$, the evaporation of the glycerol and the curing rate of PDMS are accelerated which leads to concave surface of PCML as shown in figure4(d). The virtual image can be obtained from the central region. 


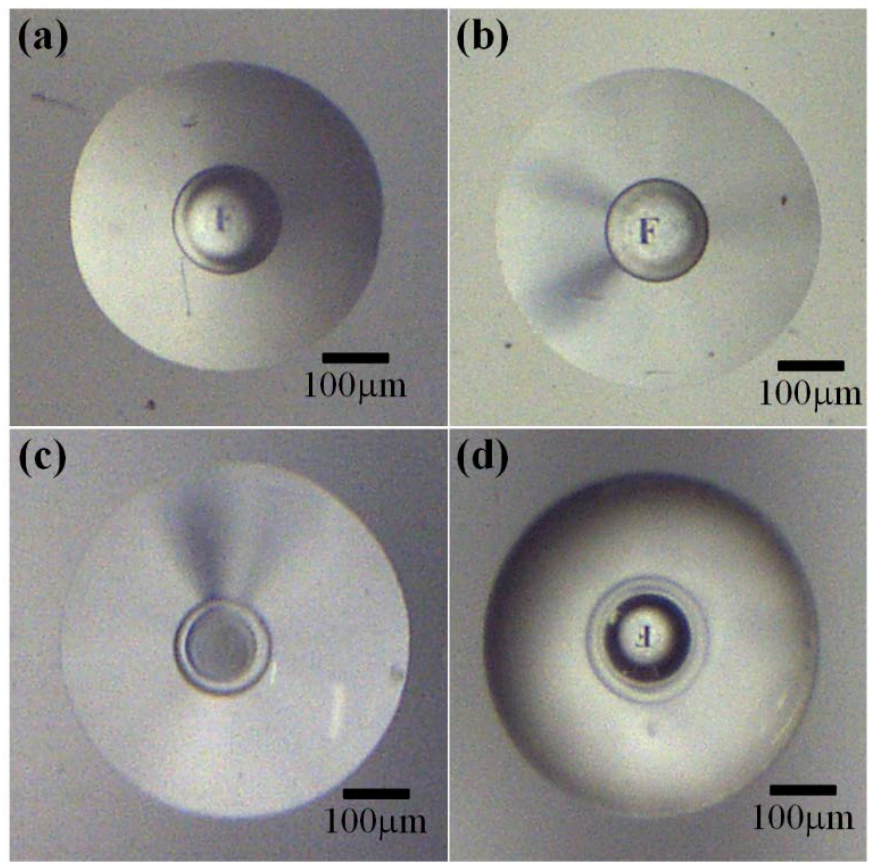

Figure 4.PCML with different curing temperature, (a) $80^{\circ} \mathrm{C}$, (b) $90^{\circ} \mathrm{C}$, (c) $100^{\circ} \mathrm{C}$ and (d) $110^{\circ} \mathrm{C}$.

Convex PCML has two lenses: the central small convex lens and out large PDMS lens (as shown in figure 2(c)), which can generate two foci. The simulation results of the two foci are shown in figure5(a) and figure5(b) respectively, which arecalculated by ASAP (simulation software) at wavelength 632.8nm. From figure5(b) we can see that a ring-shaped light distribution is obtained. The reason for the formation of this ring is that parallel light is focused on the first focal plane through the central glycerol and PDMS lenses, but light passing through the large PDMS convex lens gives rise to a ring distribution on this focal plane. The second focal spot is formed by large PDMS convex lens. Figures 5(c) and 5(d) show the real focused images, which agree well with the simulation results. From figures 5(c) we also can see a dark diffraction ring around the ring fringe, which is generated by out lens. The measured focal lengths are $453 \mu \mathrm{m}$ (first focal length) and $2592.5 \mu \mathrm{m}$ (second focal length), respectively.Because there is small amount of glycerol evaporation which results in large curvature radius of the interface between glycerol lens and the small PDMS lens under the effect of gravity. Therefore, the convex PCML can be considered as a single convex lens with half glycerol material and half PDMS material on the top of the large PDMS lens. The focal length of the planoconvex lens can be calculated by 


$$
f=\frac{R}{n-1}
$$

where $f$ is the focal length, $R$ is the curvature radius, and $n$ is the refractive index of the material ( $n=1.42$ for PDMS and n=1.48 for glycerol). Under this approximation, the focal length of the convex PCML can be calculated by

$$
F=\frac{f_{G} f_{P}}{f_{G}+f_{P}},
$$

where $F$ is the focal length of the PCML, $f_{\mathrm{G}}$ and $f_{\mathrm{P}}$ are the focal lengths of planoconvex glycerol lens and small PDMS lens. According to Eq. (2), decreasing the curvature radius of the planoconvex glycerol lens, short first focal length can be achieved. The distance between the two focal spots will increase. The first focal spot is shown in figure5(a) (simulation result) and figure5(c) (experimental result). The diameter and height of small plane-convex PDMS lens are128.5 $\mu \mathrm{m}$ and $14 \mu \mathrm{m}$, respectively. Moreover, the diameter and

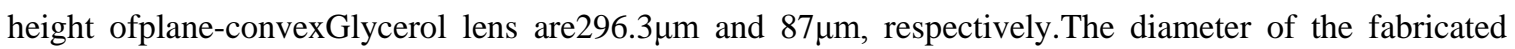
PCML is $749.7 \mu \mathrm{m}$. Therefore, theoretical focal lengths were calculated as $474.79 \mu \mathrm{m}$ (first focal length),

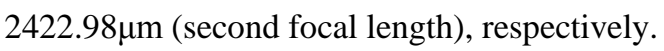
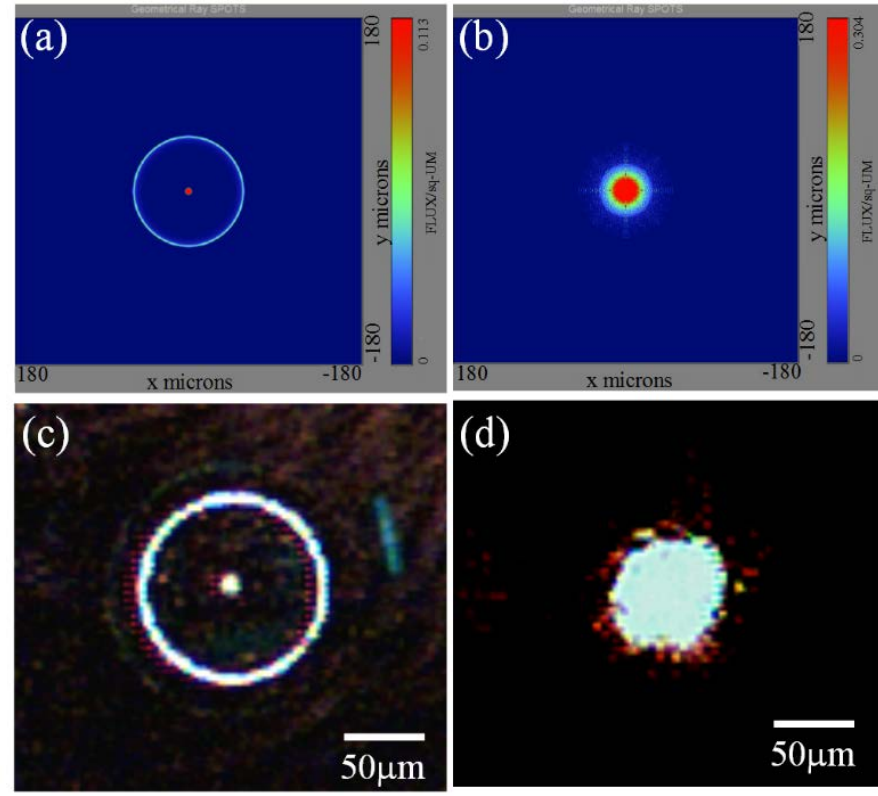

Figure5.Focusing abilityof convex PCML, (a) and (b) are the simulation results of first and second focal spot, (c) and (d) are experimental results of the first and second focal spot. 
Since the concave surface of the PCML is caused by partially evaporation of glycerol, the diameter of concave glycerol lens relates to curing temperature and curing time. Specific experiments are carried out to investigate the relationship between the concave lens diameter and the curing temperature as well as the curing time. The samples were prepared by the same glycerol and PDMS volume. When the curing temperature is less than $100^{\circ} \mathrm{C}$ under normal solidification, the glycerol rarely evaporates and it is difficult to realize concave surface. For increasing the rate of glycerol evaporation, the curing temperature should exceed $100^{\circ} \mathrm{C}$, which results in the rapid curing rate of PDMS and fast evaporation of glycerol. As shown in figure 6, the curing temperature is set as $110^{\circ} \mathrm{C}$, and with increasing of the curing time, the diameter of the concave lens increases and shows a good linearity with the curing time. When the curing time is set as30 minutes, the increasing curing temperature leads to obviously increases of the diameter. Therefore, the evaporation of the glycerol is main causes of different diameters. The higher curing temperature and longer curing time will lead to lager concave lens diameters. By adjusting the curing times and temperatures, this diametersof the concave lens can be modulated easily. Meanwhile, the concave PCMLs can also be used as the base material of convex PCMLs by filling polymer in the concave surface.

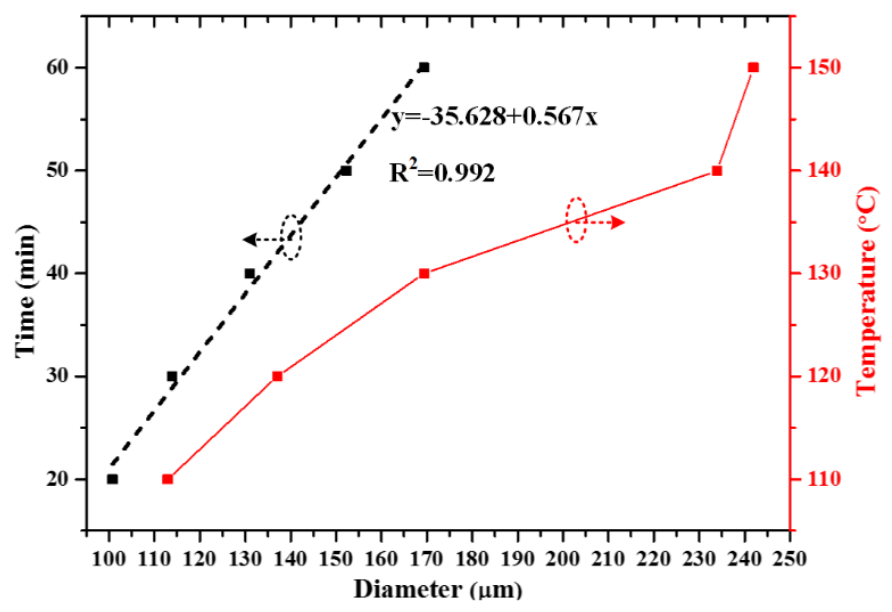

Figure 6. The relationship among the diameter, curing time and curing temperature.

\section{Conclusion}

To summarize, in this paper, we demonstrated a simple and convenient method to fabricate PCMLs array. The sandwich microstructure (PDMS-Glycerol-PDMS) can generate two different PCMLs array by changing the placing method of the samples, which are concave PCMLs array and convex PCMLs array, 
respectively, due to the evaporation of the middle glycerol. The middle glycerol lens plays an important role in modulating the top surface profile of PCML. The diameter of the concave PCML can be controlled by adjusting the solidification temperature and curing time very easily. The PCMLs array has excellent focusing ability and imaging ability. Our approach provides a simple and efficient method to large-scale production of PCMLs array for different applications in diverse optical systems.

\section{Acknowledgments}

This work is supported by National Science Foundation of China (Grant Nos. 11304064, 11304065 and 51506035);National Key Technology R\&D Program (2014BAA02B03); Special Scientific Research Found of Public Service Industry of Quality Inspection Project (201410030-03)

\section{References}

1 Lee, M. K.; Kuo, K. K. Single-step fabrication of Fresnel microlens array on sapphire substrste of flipchip gallium nitride light emitting diode by focused ion beam, Appl. Phys. Lett. 2007, 91, 05111.

2 Lu, Y.-K.; Tsai, Y.-C.; Liu, Y.-D.; Yeh, S.-M.; Lin, C.-C.; Cheng, W.-H. Asymmetric elliptic-coneshaped microlens for efficient coupling to high-power laser diodes, Opt. Express 2007, 15, 1434-1442.

3 Yang, Q.; Tong, S.; Chen, F.; Deng, Z.; Bian, H.; Du, G.; Yong, J.; Hou, X. Lens-on-lens micrstructures, Opt. Lett. 2015, 40, 5359-5362.

4 Chen, T.; Wang, T.; Wang, Z.; Zuo, T.; Wu, J.; Liu, S. Microlens fabrication using an excimer laser and the diaphragm method, Opt. Express 2009, 17, 9733-9747.

5 Zheng, C.; Hu, A.; Kihm, K. D.; Ma, Q.; Li, R.; Chen, T.; Duley, W. W. Femtosecond laser fabrication of cavity microball lens (CMBL) inside a PMMA substrate for super-wide angle imaging, Small 2015, 11, 3007-3016.

6 Oder, T. N.; Shakya, J.; Lin, J. Y.; Jiang, H. X. Nitride microlens arrays for blue and ultraviolet wavelength applications, Appl. Phys. Lett. 2003, 82, 3692-3694.

7 Ou, Y.; Yang, Q.; Chen, F.; Deng, Z. F.; Du, G. Q.; Wang, J. H.; Bian, H.; Yong, J. L.; Hou, X. Direct Fabrication of Microlens Arrays on PMMA With Laser-Induced Strcutural Modification, IEEE Photon. Technol. Lett. 2015, 27, 2253-2256.

8 Wu, M. H.; Park, C.; Whitesides, G. M. Fabrication of twodimensional arrays of microlenses and their applications in photolithography, Langmuir 2002, 18, 9312-9318.

9 Bonaccurso, E.; Butt, H.-J.; Hankeln, B.; Niesenhaus, B.; K. Graf Fabrication of microvessels and microlenses from polymers by solvent droplets, Appl. Phys. Lett. 2005, 86, 124101.

10 Aymerich, M.; Nieto, D.; Flores-Arias, M. T. Laser-based surface multistructuring using optical elements and the Talbot effect, Opt. Express 2015, 23 24369-24382. 
11 Deng, Z. F.; Yang, Q.; Chen, F.; Meng, X. W.; Bian, H.; Yong, J. L.; Shan, C.; Hou, X. Fabrication of large-area concave microlens array on silicon by femtosecond laser micromachining, Opt. Lett. 2015, 40, 1928-1931.

12 Xia, Z.; Li, Y.; Han, Y.; Ge, M.; Ye, J.; Deng, D.; Wang, B.; Gao, J.; Qu, S. Taper array in silica glass for beam splitting, Optical Materials 2016, 53, 6-9.

13 Lu, D.-X.; Zhang, Y.-L.; Han, D.-D.; Wang, H.; Xia, H.; Chen, Q.-D.; Dingc, H.; Sun, H.-B. Solventtunable PDMS microlens fabricated by femtosecond laser direct writing, J. Mater. Chem. C 2015, 3, 1751-1756.

14 Ou, Y.; Yang, Q.; Chen, F.; Deng, Z.; Du, G.; Wang, J.; Bian, H.; Yong, J.; Hou, X. Direct Fabrication of Microlens Arrays on PMMA With Laser-Induced Structural Modification, IEEE Photon. Technol. Lett. 2015, 27, 2253-2256.

15 Komma, Y.; Tanaka, Y.; Urairi, K.; Nishino, S.; Mizuno, S. Dual Focus Optical Head with a Hologram-Integrated Lens, Jpn. J. Appl. Phys. 1997, 36, 474.

16 Lin, Y.-J.; Chiu, Y.; Shih, H.-F.; Chiou, J.-C. Fabrication and verification of a small-form-factor bluelight optical pickup head with holographic optical element, Lightwave Technology 2012, 30, 38-42.

17 Golub, M. A.; Shurman, V.; Grossinger, I. Extended focus diffractive optical element for Gaussian laser beams, Appl. Opt. 2006, 45, 144-150.

18 Leung, H. M.; Zhou, G.; Yu, H.; Chau, F. S.; Kumar, A. S. Liquid tunable double-focus lens fabricated with diamond cutting and soft lithography, Appl. Opt. 2009, 48, 5733-5740. 